\title{
Condensation of Malonic Diethyl Ester with Acrylamide
}

\author{
Gagik Torosyan*, Nelli Hovhannisyan and Dezi Hovhannisyan \\ Department of Chembio and Environmental Technologies, National Polytechnic University of Armenia, Republic of Armenia
}

*Corresponding author: Gagik Torosyan, Department of Chembio and Environmental Technologies, National Polytechnic University of Armenia, Republic of Armenia.

To Cite This Article: Gagik Torosyan, Condensation of Malonic Diethyl Ester with Acrylamide. 2020 - 7(3). AJBSR.MS.ID.001157.

DOI: $10.34297 /$ AJBSR.2020.07.001157.

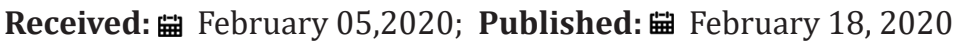

\begin{abstract}
The purposeful synthesis of polyfunctional organic substances is an important task in organic chemistry, as well as for solving practical problems in technologies of fine organic chemistry and biochemistry. Here presents the results of studies on the synthesis of polyfunctional substances as possible ligand based on with malonic acid diethyl ester. The condensation of malonic acid diethyl ether with acrylamide in phase transfer catalysis conditions leads to new derivative of malonic acid-1,1-dicarbethoxy-3-carbamoilpropane. The formation of mentioned compound has been followed by the method of UV spectrometry. The complexatation of the received substance investigated in relation to iron ions also.
\end{abstract}

Keywords: Acrylamide, Organic substances, Malonic Acid Diethyl Ester, UV spectrometry, Trimethylcetyl Ammonium Bromide, Lauryl Sulfonate, Phase Transfer Catalytic, Trimethylcetyl Ammonium Chloride, Iron Ion Extraction, Deionized water

\section{Introduction}

Synthesis of metal complex compounds of a number of vital elements based for instance as acetyl-carbethoxy-carbamoil containing group organic molecules is important task in chemistry, especially in metal-organic chemistry and chemical technology [1]. The resulting compounds are widely used as physiologically active compounds with a wide range of capabilities, as well as in manufacture of paints. Such compounds can also be used in wastewater treatment from metal ions. Previously it has been mentioned that acrylamide enters Michael condensation with acetoacetic ether under Phase Transfer Catalytic (PTC) conditions "solid phase-liquid" [1]. It was established also that an increase in the surface activity of phase transfer catalyst contributes to the yield of the product of addition [2]. It had been assumed that the condensation takes place either in the aqueous phase or at the boundary of the separated phases. This interaction was carried out at room temperature $\left(25-30^{\circ} \mathrm{C}\right)$ an as the best PTC catalysts had been proposed trimethylcetyl ammonium bromide and Lauryl sulfonate.
In this article presents the results of studies on the synthesis of a polyfunctional substances as possible ligand based on with Malonic Acid Diethyl Ester (MADEE), where three atomic groups can functioned as donor atoms in the coordination complex. To achieve this, we used a method previously developed in our laboratory for the synthesis of such compounds by the reaction of Michael for Acrylamide (AA) with ethyl ether of acetatoacetic acid [1]. In the present work, we used MDEE as an alkylating agent AA, weaker C-H acid pKa - 10.7, than acetoacetic acid ethyl ester/pKa 9.5/ acid. Trimethylcetyl Ammonium Chloride (TMCACh) was used as a phase transfer catalyst [2].

\section{Experimental Part}

\section{Equipment and Chemicals}

Malonic Acid Diethyl Ester (MADEE) and Acrylamide (AA) was supplied by the National Polytechnic University of Armenia supplier. The structure of the obtained compounds is confirmed by PMR spectroscopy, IR and mass spectrometry. IR spectra were recorded on a SPECORD M-80 IR spectrometer. The reaction progress and 
purity of the compounds were monitored by TLC on SilufolUV-254 plates in an eluent was benzene-ethanol (2:1), and the display was performed using iodine vapor. The maximum absorption of MDEE in water without $\mathrm{KOH}$ is observed at dmax $=250 \mathrm{~nm}$, and in the presence of $\mathrm{KOH}$ after 20 minutes stirring at $20 \mathrm{oC}-270 \mathrm{~nm}$. This can be explained by the formation of the keto-enol form of diketone with $\mathrm{KOH}$, in the absorption spectrum of which the maximum corresponds to this region.

1H NMR spectra were recorded on a Varian MERCURY-300 NMR spectrometer $(300 \mathrm{MHz})$, in DMSO-d6, internal standardHMDS. The working frequency on protons is $300.077 \mathrm{MHz}$, on the $13 \mathrm{C}$ nucleus-75.46MHz, chemical shifts are given in ppm regarding TMS. The study of 1,1-dicarbethoxy-3-carbamoilpropane presence in aqueous solution was carried out using Sciex Triple Quad ${ }^{\mathrm{TM}} 4500$ Quadruple Mass Spectrometer. The sample dilution was carried out using HPLC Grade Methanol and Deionized water supplied by Carl Roth, Germany. Formic Acid (98\% ACS grade) Carl Roth (Germany). The instrument and chemicals were supplied by Darmantest Laboratories, Armenia.

\section{The synthesis of 1,1-dicarbethoxycarbamoilpropane}

In a flask equipped with a stirrer, reflux condenser and a dropping funnel at $20^{\circ} \mathrm{C}$, a mixture of $9.12 \mathrm{~g}(57.6 \mathrm{mmol})$ of MADEE, $4.8 \mathrm{ml}$ of a $10 \mathrm{~N}$ aqueous solution of $\mathrm{KOH}(57.6 \mathrm{mmol})$ and $2.4 \mathrm{~mol}$ of TMCACh were stirred. Within 20 minutes a solution of $3.4 \mathrm{~g}$ AA in $25 \mathrm{ml}$ of water $(57.6 \mathrm{mmol}$ ) was added drop wise to the mixture. The reaction mixture was stirred for 2 hours, extracted with benzene, and then an aqueous layer was acidified with $5.9 \mathrm{ml}$ of $30 \% \mathrm{HCl}$ and extracted again with benzene. The benzene extracts were combined and dried over $\mathrm{MgSO}_{4}$ overnight, after which benzene was distilled off.

It had received $5.4 \mathrm{~g}(24 \mathrm{mmol})$ of 1,1-dicarbethoxy-3carbamoilpropane with yield 50\%. Rf 0.75. Found, \%: C-52.30, H-7.02, N- 5.95; $\mathrm{C}_{10} \mathrm{H}_{17} \mathrm{NO}_{5}$. Calculated, \%: C 51.95; H 7.36; N 6. 06. IR spectrum, , cm ${ }^{-1}: 3420,3300\left\{\right.$ vas ,vs $\left(\mathrm{NH}_{2}\right), 2965,2870\left\{\mathrm{v}\left(\mathrm{CH}_{3}\right)\right.$ \}$, 2895\{\mathrm{v}(\mathrm{CH})\}, 1735\{\mathrm{v}(\mathrm{CO}) \mathrm{C}-\mathrm{C}(\mathrm{CO})-\mathrm{O}\}, 1680\{\mathrm{v}(\mathrm{CO}) \mathrm{C}-\mathrm{C}(\mathrm{O})-$ $\mathrm{NH}_{2}, 1640\{\delta(\mathrm{NH})\}, 1465,1380\left\{\delta\left(\mathrm{CH}_{3}\right)\right\}, 1140,1030\{\delta(\mathrm{C}-\mathrm{O}-\mathrm{C})$ \}, $720\left\{\delta\left(\mathrm{CH}_{2}\right)\right\} .1 \mathrm{H}$ NMR spectrum $\left(\mathrm{CDCl}_{3}\right), \delta, \mathrm{ppm}: 1.25 \mathrm{t}(6 \mathrm{H}$, $\left.-\mathrm{OCH}_{2} \mathrm{CH}_{3}, \mathrm{~J} 5.2 \mathrm{~Hz}\right), 2.15-2.35 \mathrm{~m}\left(4 \mathrm{H},-\mathrm{CH}-\mathrm{CH}_{2} \mathrm{CH}_{2} \mathrm{C}(\mathrm{O}) \mathrm{NH}_{2}\right), 3.15 \mathrm{~m}$ $(1 \mathrm{H}, \mathrm{C}(0)-\mathrm{CH}-\mathrm{C}(\mathrm{O}) \mathrm{J} 6,4 \mathrm{~Hz}), 4.1 \mathrm{~m}\left(4 \mathrm{H}, \mathrm{CH}_{3} \mathrm{CH}_{2}-\mathrm{O}-\right.$ ), 7,04s ( $2 \mathrm{H}$, $\mathrm{NH}_{2}$ ). In the mass spectra of 1,1-dicarbethoxy-3-carbamoilpropane there are quite intense peak of molecular ion, the most common and consistent ions were observed at $\mathrm{m} / \mathrm{e} 231.54$.

\section{Iron Ion Extraction}

$10 \mathrm{ml}$ of a $0.01 \mathrm{M}$ benzene solution of 1,1-dicarbethoxy-3carbamoilpropane and $1 \mathrm{ml}$ of a $0.001 \mathrm{M}$ aqueous solution of $\mathrm{FeCl}_{3}$ were placed in a separatory funnel, $5 \mathrm{ml}$ of a $1 \mathrm{M} \mathrm{KNO}_{3}$ solution, $4 \mathrm{ml}$ of $\mathrm{H}_{2} \mathrm{O}$ were added. The mixture was stirred for 30 minutes, sedimented for 15 minutes. When moving, there was a color of the aqueous solution changed from light yellow to light lilac. The isolated aqueous layer was treated with ammonium rhodanite and a solution of amyl alcohol with ether, it was stained with a blood red color, which was compared with the color of the alcohol-ether extract obtained with treating the initial solution $(1 \mathrm{ml}$ of $0.001 \mathrm{M}$ $\mathrm{FeCl}_{3}$ and $9 \mathrm{ml}$ of $\mathrm{H}_{2} \mathrm{O}$ ) under similar conditions [3].

\section{Results and Discussion}

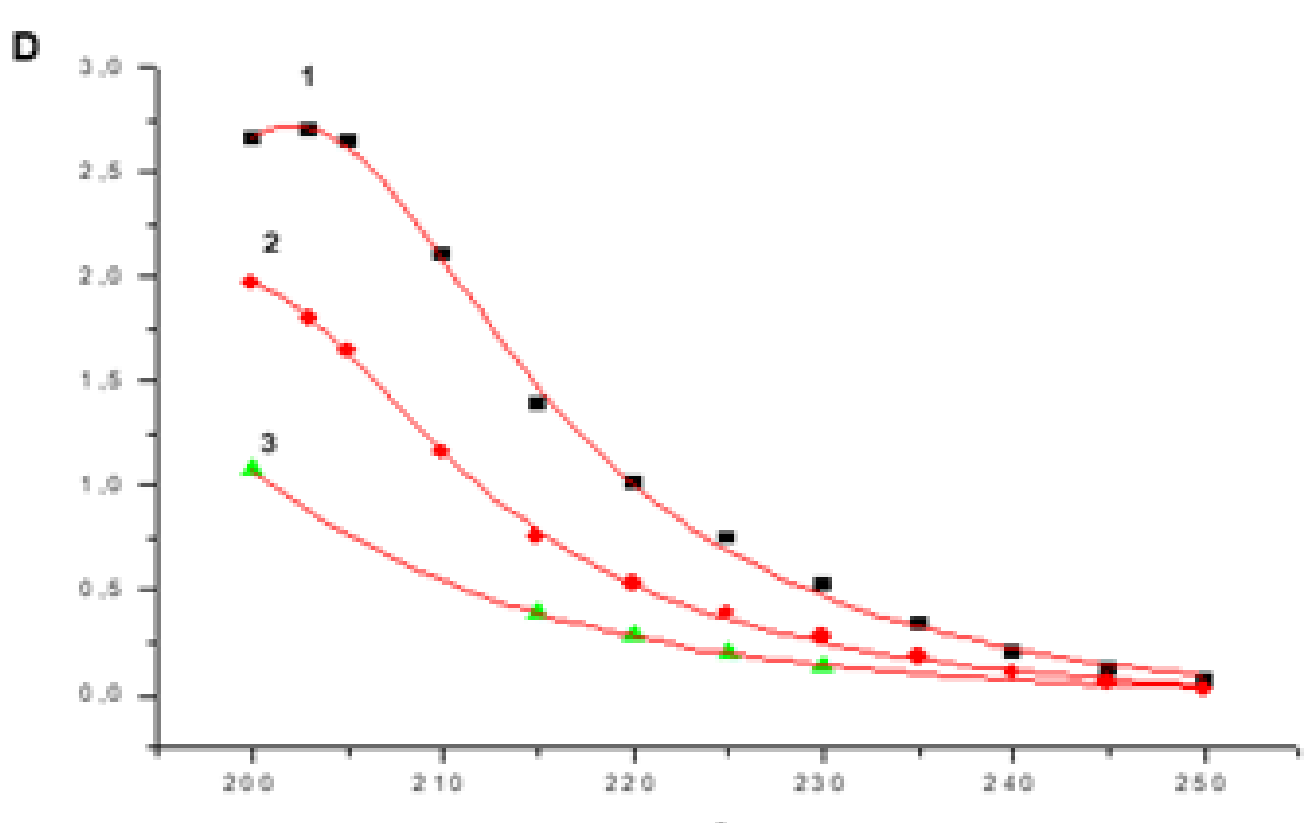

$\lambda$

Figure 1: The dependence of $D-\lambda$, from $[A A] 0=3.2 \times 10^{-4}(1), 1.6 \times 10^{-4}(2), 0.8 \times 10^{-4}(3)$ 


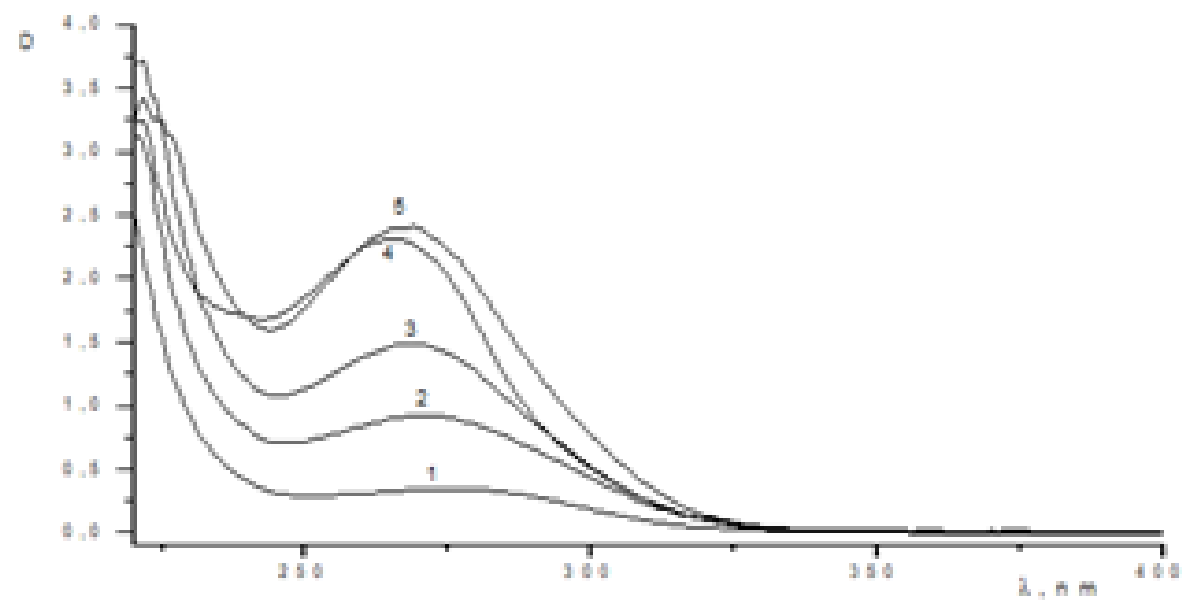

Figure 2: UV absorption spectrum of MADEE $+\mathrm{KOH}$ system in water at, $\mathrm{t}=30^{\circ} \mathrm{C}[\mathrm{MDEE}](\mathrm{mol} / \mathrm{L})=5.0 \times 10^{-3}(1), 3.0 \times 10^{-2}(2), 2.0 \times 10^{-2}(3)$, $1.5 \times 10^{-2}(4), 1.3 \times 10^{-2}(5)$.

The interaction`s progress was monitored according to the ultraviolet measurement of the reaction mixture with the resulting reaction product. In addition, measurements were made for $\mathrm{AA}+$ $\mathrm{KOH}$ and MADEE + KOH systems to determine calibration curves. Initially, UV studies and ASPECT PLUS software generated electron absorption diagrams of acrylamide and the reaction system at various stages of the reaction (Figure 1). UV studies showed that the maximum absorption of $A A$ in water is $\operatorname{lmax}=220 \mathrm{~nm}$. The maximum absorption of in water with the MADEE + KOH system is $\operatorname{lmax}=250 \mathrm{~nm}$, however, after 20 minutes of stirring at $\mathrm{t}=30^{\circ} \mathrm{C}$, the maximum changes to $\operatorname{lmax}=270 \mathrm{~nm}$. this can be explained by the interaction of MADEE with $\mathrm{KOH}$, as a result of which associates are obtained whose absorption spectrum corresponds to this region at $[\mathrm{MADEE}]>10^{-1} \mathrm{~mol} / \mathrm{L} \mathrm{l} \max =355 \mathrm{~nm}$. Then the calibration curve D-[MADEE] was displayed (Figure 2).
This can be explained by the interaction of $\mathrm{C}$-acid with $\mathrm{KOH}$, as a result of which products are obtained whose maximum absorption spectrum corresponds to this region. The reaction rate was monitored by measuring the optical density of the MDEE $+\mathrm{KOH}+\mathrm{AA}$ system at $\operatorname{lmax}=270 \mathrm{~nm}$. Samples taken over time were diluted to $10^{-3} \mathrm{~mol} / \mathrm{L}$, calculated on the concentration of MADEE, the optical density was measured. To switch from optical density to concentration, optical density was measured at various concentrations (Figure 3). Then the calibration curve D- [MADEE] was displayed (Figure 2). The reaction rate was monitored by measuring the optical density of the MADEE + $\mathrm{KOH}+$ AA system. Samples taken over time were diluted to $10^{-3} \mathrm{~mol} / \mathrm{L}$, calculated on the concentration of MADEE, and the optical density was measured. To switch from optical density to concentration, the optical density was measured at various concentrations. Then the calibration curve D-[MADEE] was derived.

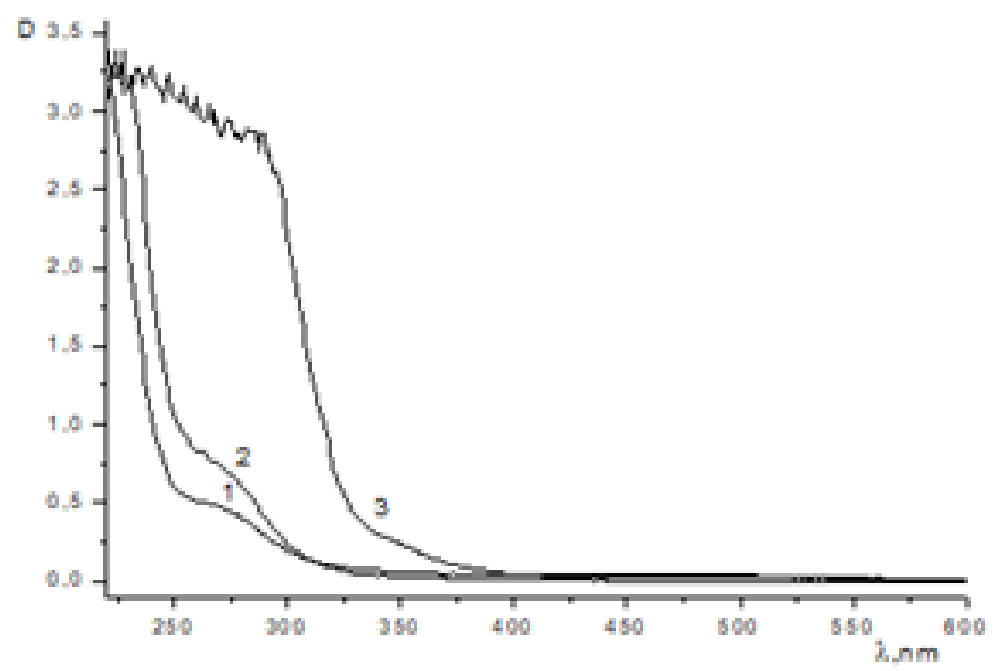

Figure 3: UV absorption spectrum of the MADEE $+\mathrm{KOH}+\mathrm{AA}$ system in water at $\mathrm{t}=20^{\circ} \mathrm{C}[\mathrm{MADEE}](\mathrm{mol} / \mathrm{L})=0.752 \times 10-3(1), 1.52 \times 10-3(2)$, $1.55 \times 10-2(3)$. 
When $[\mathrm{MADEE}] \approx[\mathrm{AA}]$, the reaction rate constant can be determined from the dependence 1/[MADEE]-1/[MADEE] $0=\mathrm{kt}$,
(Figure $4 \& 5$ ). With this method of calculation, the value of the rate constant is also equal to $\mathrm{k}=1.9 \times 10^{-4} \mathrm{l} / \mathrm{mol} \mathrm{sec}$.

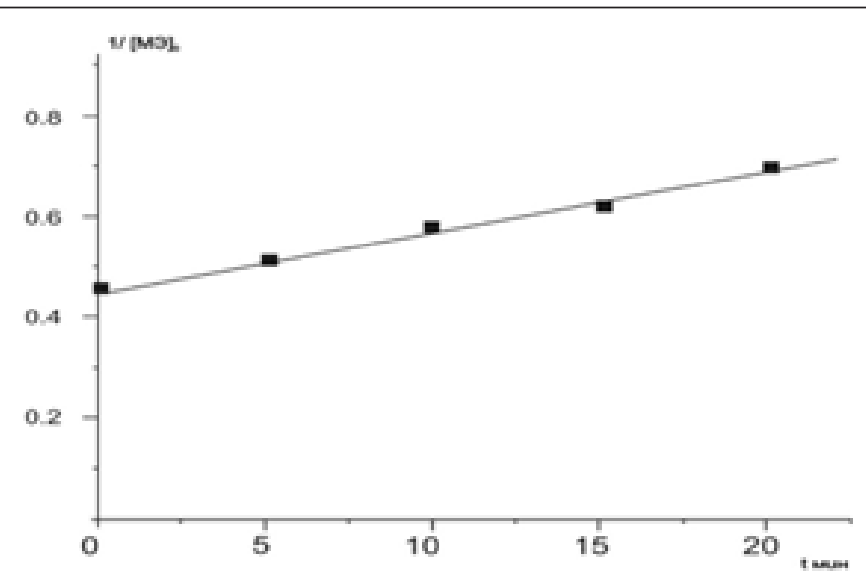

Figure 4: Dependence 1/[MADEE] 0-t in the reaction MADEE+AA.

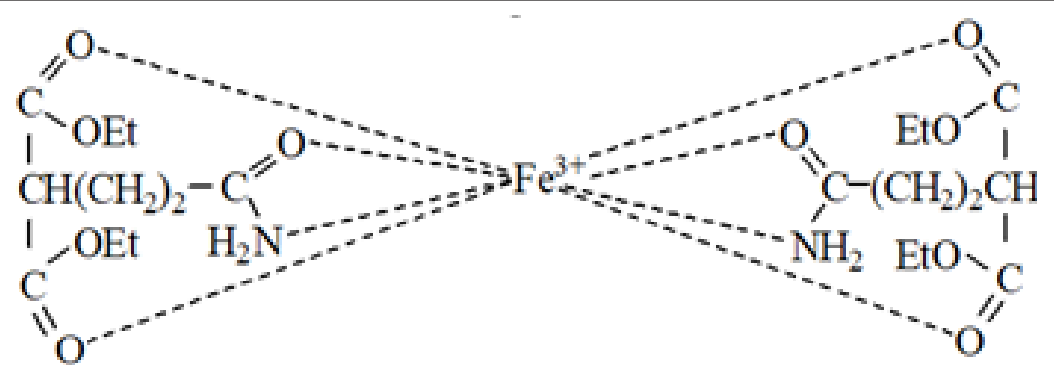

Figure 5: Formation of an iron complex with two complexing agent molecules.

The resulting compounds differ in intense color, which varies depending on the concentration of the organic solution of the MADEE derivative. It should be noted that, by-products of the polymerization of acrylamide (AA) or other MADEE transformations are formed with a small yields, which were not considered in this work. The synthesis of metal complex compounds of vital elements with ligands from a number of polyfunctional organic molecules containing acetyl-carbethoxy-carbamoyl groups is known [3]. Such compounds are widely used as physiologically active substances with a wide range of capabilities, as auxiliary agents in the agricultural industry, in the manufacture of dye materials, and can be used also in the treatment of wastewater from metal ions.

1,1-dicarbethoxy-3-carbamoilpropane can be used as a ligand also. To this goal the interaction of the newly obtained compound with a ferric ion was studied. The resulting complex compound, in all probability a chelate formation, is characterized with a lower color intensity compared to the analogous derivative of acet oacetic ester [3]. Here are we did not set ourselves the task of a detailed study of the stoichiometry of possible complexes, their stability, and other properties, confining ourselves only to proving the very fact of their formation. Iron (III) chloride solution was extracted with benzene solution of the aforementioned 3-carbamoylpropane derivative to prove complexation. Since the iron salt in benzene is practically insoluble, a decrease in the concentration of its aqueous solutions after such extraction is possible only in the case of the formation of complexe with organic ligand.

The initial aqueous solutions of salts had a concentration of $\sim 0.001 \mathrm{~mol} / \mathrm{L}$, i.e., for iron $\sim 5.585-2 \mathrm{~g} / \mathrm{L}$; the concentration of the benzene solution of the extracting agent was taken from the calculation of the known excess of the alleged complexation agent (with equal volumes of phases) equal to $0.01 \mathrm{~mol} / \mathrm{L}$. The initial

salt concentration ( $C_{\text {initial }}{ }^{0}$ ) was determined by the extractionphotometric method using, as the determined form, complexes of iron with ammonium rhodanite and a mixture of amyl alcohol with diethyl ether ( $3: 1$ by volume) as an extractant [4]; the concentration of the aqueous phase was also determined after extraction with benzene of the resulting complexes with derivatives of $\mathrm{CH}$-acids (

$$
C_{\text {after }}{ }^{f} \text { ). }
$$

The results were evaluated by the distribution coefficient between phases; if the initial volumes of the phases are equal and taking them unchanged after extraction, we can assume that the 
iron concentration $\left(\mathrm{C}_{\text {org }}{ }^{0}\right)$ in the organic phase is:

$$
C_{\text {org }}^{f}=C_{\text {initial }}^{0}-C_{\text {after }}{ }^{f}
$$

Then the mass distribution coefficient of the corresponding ion $\mathrm{Fe}^{+3}$ will be equal to:

$$
\varphi=\left(C_{\text {initial }}^{0}-C_{\text {after }}^{f}\right) / C_{\text {after }}{ }^{f}
$$

During the formation of a complex of 1,1-dicarbetoxy-3carbamoylpropane with iron (III), the following data were

obtained: $\quad C_{\text {initial }}{ }^{0} \mathrm{~g} / \mathrm{l}-5.7 \times 10^{-2}, \quad C_{\text {after }}{ }^{f} \mathrm{~g} / \mathrm{l}-3.3 \times 10^{-6}$, mass distribution coefficient $-\varphi$ 17271. From the obtained data it follows that the compound studied forms a complex with iron, while the residual concentration of iron in the aqueous phase is very small and practically does not change from experiment to experiment. This result is explained by the fact that, in the absence of a priori information on stoichiometric relationships in the proposed complexes, and not setting the task of obtaining quantitative characteristics of the interaction stoichiometry. We assume that the probable formation of an iron complex with two complexing agent molecules: The complexing properties of the compounds under discussion can find various applications, for example, in the processes of extracting metals from galvanic waste, non-ferrous metal mining, etc.

\section{Conclusion}

The condensation of malonic acid diethyl ether with acrylamide in phase transfer catalysis conditions leads to new derivative of malonic acid-1,1-dicarbethoxy-3-carbamoilpropane. The obtained compound shows the complexation capability in relation to iron ions.

\section{References}

1. Torosyan GH, Harutyunyan AL, Isakova LA, Beylerian NM, Chobanyan JN, et al. (2007) Reaction of AAEE with acryl \& methyl acryl amides in conditions of inverse PTC. Effect of surfactant's type. Oxidation communication 10(3): 548-552.

2. Torosyan GH, Harutyunyan AL, Isakova LA, Chobanyan JN, Simonyan GS (2007) The interaction of acetoacetic acid ester with acrylic and methylacrylamides in the presence Quat, Proceedings of the Universities of the Russian Federation, a series of chemistry and chemical technology 50(11): 28-30.

3. Torosyan GH, Aleksanyan AR, Isakova LA, Simonyan GS (1967) Synthesis and chelation of acetoacetic ester derivative.

4. Handbook of a chemistry: Chemistry 4: 920. 